\title{
The reliability of dual rehabilitative ultrasound imaging measurements for muscle co-activation
}

\author{
Joohee Hahn ${ }^{a}$, Hyun-Geun Ha ${ }^{b}$, Hwang-Jae Lee ${ }^{c}$, Seungyeop Lim ${ }^{a}$, Wan-hee Lee ${ }^{d}$ \\ ${ }^{a}$ Department of Physical Therapy, Health and Welfare Graduate School, Sahmyook University, Seoul, Republic of Korea \\ ${ }^{b}$ Department of Physical Therapy, Health \& Health Care, Namseoul University, Cheonan, Republic of Korea \\ 'Department of Health Sciences and Technology, Samsung Advanced Institute for Health Sciences \& Technology, Samsung Medical Center, \\ Sungkyunkwan University, Seoul, Republic of Korea \\ ${ }^{\mathrm{d} D e p a r t m e n t ~ o f ~ P h y s i c a l ~ T h e r a p y, ~ C o l l e g e ~ o f ~ H e a l t h ~ S c i e n c e ~ a n d ~ S o c i a l ~ W e l f a r e, ~ S a h m y o o k ~ U n i v e r s i t y, ~ S e o u l, ~ R e p u b l i c ~ o f ~ K o r e a ~}$
}

\begin{abstract}
Objective: The aim of this study was to determine the intra-rater and inter-rater reliability of the dual rehabilitative ultrasound imaging (D-RUSI) when simultaneously measuring muscle thickness changes at rest and during co-contraction of the biceps brachii (BB) and triceps brachii (TB).

Design: Cross-sectional study.

Methods: This study included 36 healthy participants ( 23 men, 13 women). The participants sat on a chair in a comfortable position with a cushion placed under their elbow to maintain a 90-degree elbow flexion angle. The muscle thickness of the biceps brachii and triceps brachii was measured twice using the D-RUSI by two examiners during resting and co-contraction states. One week later, the same procedure was performed once again.

Results: The intra-class correlation coefficients (ICCs) for intra-rater reliability ranged from 0.887 to 0.989 and the confidence interval was within an acceptable range of 0.778 to 0.994 . The standard error of measurement (SEM) values ranged from 0.303 to 0.866 and the minimal detectable change (MDC) values ranged from 0.84 to 2.40 . The ICCs for inter-rater reliability ranged from 0.758 to 0.925 . The SEM values ranged from 0.702 to 1.486 and the MDC values ranged from 1.95 to 4.12 .

Conclusions: The use of the D-RUSI of the BB muscle had a very good intra-rater reliability and very good inter-rater reliability at the resting state, and a, good inter-rater reliability at the co-activation state. ICC values showed very good intra-reliability and inter-reliability for the TB muscle. the D-RUSI is a useful tool for simultaneously measuring the thickness of two muscles when the $\mathrm{BB}$ is an agonist and the TB is an antagonist during co-activation of the upper arm.
\end{abstract}

Key Words: Humerus, Muscle contraction, Reproducibility of results, Ultrasonography

\section{Introduction}

Muscle strength is a fundamental aspect of the performance of everyday tasks [1]. It is essential for health, functional ability, and quality of life, and varies greatly according to the circumstances of growth, aging, and lifestyle [2]. In particular, age and disease have been shown to reduce muscle mass and strength, which can lead to adverse physiologic changes and functional limitations [3]. The action of large muscle groups, especially in the upper extremities, is necessary for daily tasks such as carrying, pulling and pushing [1]. Previous research has shown that upper extremity muscle weakness can limit activities of daily living, particularly in the use of instruments [4]. The muscle strength of the upper limbs must be supported to enable skilled hand function, and the muscles of the upper limb are necessary to properly adjust the other joints of the arms, provide hand stability, and perform delicate tasks. Indeed, a decrease in up-

Received: 12 June, 2017 Revised: 7 September, 2017 Accepted: 8 September, 2017

Corresponding author: Wan-hee Lee

Department of Physical Therapy, College of Health Science and Social Welfare, Sahmyook University, 815 Hwarang-ro, Nowon-gu, Seoul 01795, Republic of Korea

Tel: 82-2-3399-1633 Fax: 82-2-3399-1639 E-mail: whlee@syu.ac.kr

(c) This is an Open-Access article distributed under the terms of the Creative Commons Attribution Non-Commercial License (http://creativecommons.org/licens es/by-nc/4.0) which permits unrestricted non-commercial use, distribution, and reproduction in any medium, provided the original work is properly cited.

Copyright $\odot 2017$ Korean Academy of Physical Therapy Rehabilitation Science 
per limb strength results in an insufficient stability in the proximal arm, which may limit grip strength [1]. Clearly, upper body strength management is a critical determinant of quality of life.

Most strength training methods apply an external force toward the body segment end by lifting heavy equipment to provide resistance to the contraction muscles. However, isometric training has been proposed as a means of strength training using paired contractions of the agonists and antagonists without joint movement [5,6]. In fact, co-activation is the simultaneous activation of the agonist and antagonist muscle groups around the joints. The torque produced during the maximum voluntary contraction corresponds to the algebraic sum of the agonist and antagonist torques and is influenced by the activation of both the agonists and antagonists.

This co-activation is important for joint stabilization, the uniform distribution of pressure within the joint capsule, and prevention of bone displacement [7]. In the case of co-contraction of muscles, only electromyography has been used to measure muscle activity in real time, and the research on rehabilitative ultrasound imaging (RUSI) has only evaluated the measurement of muscle thickness before and after co-contraction training $[8,9]$.

RUSI is a potentially useful tool for the evaluation and treatment of muscular dysfunctions, particularly for measuring the morphological characteristics of muscles and enabling direct observation of muscle contractions for biofeedback [10]. However, since there is only one probe to measure the image, only one muscle can be measured in real time, which is a disadvantage. Recently, the dual RUSI (D-RUSI),

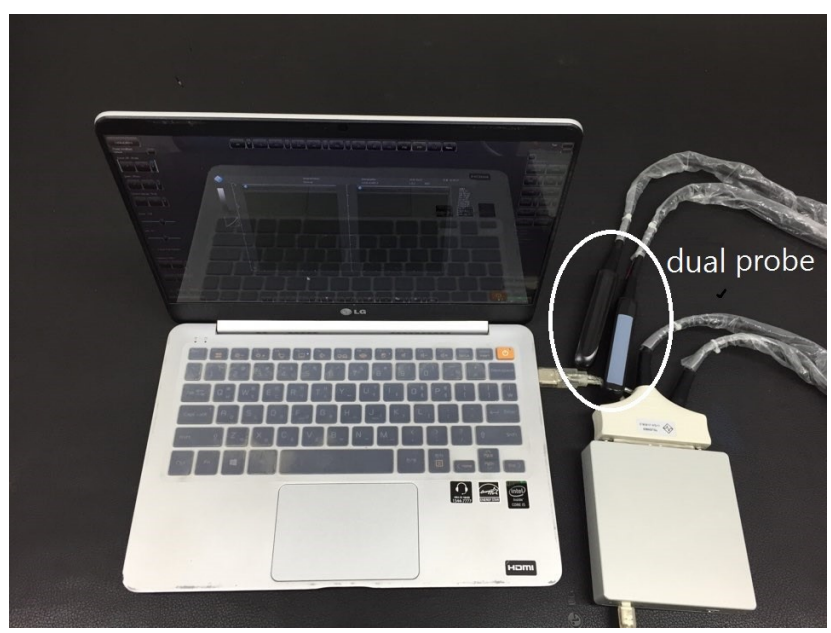

Figure 1. Dual rehabilitative ultrasound imaging. a device capable of simultaneously measuring the action of two muscles in real time was developed, with two probes and two measurement zones displayed on one screen. The purpose of the present study was to determine the reliability of the D-RUSI by simultaneously measuring muscle thickness changes during co-contraction of the biceps and triceps.

\section{Methods}

\section{Participants}

Thirty-six healthy individuals (23 men, 13 women) voluntarily agreed to participate into the study. All healthy participants were aged in their 20 s and 30 s and had a full active range of motion. Participants had no history of skeletal muscle pain or disease within the past 3 months. The exclusion criteria was musculoskeletal or neuromuscular disorders in the upper extremity, pregnancy, and body mass index (BMI) $>30 \mathrm{~kg} / \mathrm{m}^{2}$. The study was approved by the Institutional Review Board of Sahmyook University (IRB No. 21040781-AB-N-01-2016085HR) in Seoul. All participants were given a written explanatory statement regarding the procedure and the risks involved in this study and then asked to sign a consent form if they were willing to participate.

\section{Procedures}

Prior to the experiment, the anthropometric variables of the participants were measured by one skilled rater. The upper arm circumference was measured using a tape measure at the thickest part of the upper arm muscle belly.

The ultrasonography device (dual-MicrUs EXT; Telemed, Vilnius, Lithuania) used in the study was an imaging unit set in B mode with 7.5 MHz dual linear array transducer (Figures $1,2)$. The muscle thickness was measured during co-con-

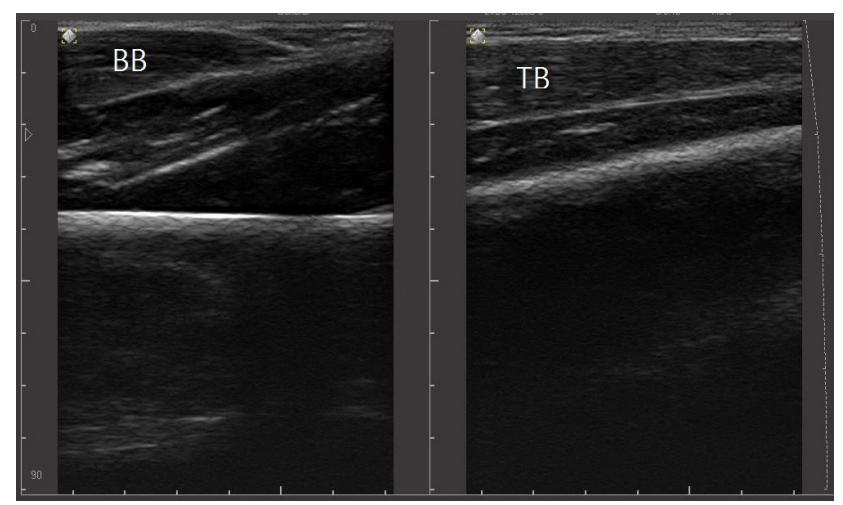

Figure 2. Simultaneously measuring two muscles in real time. BB: biceps brachii, TB: triceps brachii. 
traction of the biceps brachii (BB) and triceps brachii (TB) by an experienced physiotherapist. Two raters performed the measurements for the reliability study. Measurements were performed two times at rest and two times at maximal voluntary contraction (MVC) per rater, with one rater measuring first, followed by another rater one minute later. The mean values of the two measurements were used in the analyses. In addition, the same experiment was performed one week later for the analysis of test-retest reliability. When measuring the muscle structure, ultrasound gel was used between the transducer and the skin to increase the area of contact and to minimize the need for inadequate inward probe pressure [11]. The participants sat on a chair in a comfortable position, with a cushion placed under their elbow to maintain a 90-degree elbow flexion angle. The thickness of the $\mathrm{BB}$ and TB was measured by placing the transducer vertically and on the anterior and posterior aspect of the upper arm proximal to the crease of the elbow $[12,13]$. In the resting state, muscle thickness was measured first. Then, the isometric contraction was induced to measure the muscle thickness during co-activation of the BB and TB. The MVC value was measured using a digital manual muscle tester (Power Track II; JTECH Medical Industries, Inc., Midvale, UT, USA) while measuring the thickness at the time of muscle contraction. The measured MVC value was used to make the muscle contraction constant during repeated measurements.

We analyzed both the imaging and calculated measurements of muscle thickness using Echo Wave II 3.6.1.b (Telemed).

\section{Statistical analysis}

The analysis was performed using IBM SPSS Statistics ver. 18.0 (IBM Co., Armonk, NY, USA), and the statistical level of significance was set at 0.05 . Comparisons of the participants' general characteristics were performed using the independent t-test. To assess the reliability of the D-RUSI, the intra-class correlation coefficient (ICC) was calculated for the muscle thickness of the $\mathrm{BB}$ and $\mathrm{TB}$ at rest and during co-activation. ICCs of the type $(3,1)$ were used to assess the reliability of each parameter. ICC values were categorized as follows: poor, $\leq 0.20$; fair, 0.21-0.40; moderate, 0.410.60 ; good, $0.61-0.80$; and very good, $\geq 0.81$ [14]. In order to evaluate the accuracy of the measurement, the standard error of the measurement (SEM) was calculated as the standard deviation $\times \sqrt{[1-I C C}]$. The minimal detectable change (MDC) was calculated as $1.96 \times \mathrm{SEM} \times \sqrt{2}$, which is the mini- mum thickness change when $95 \%$ confidence interval (CI) is assumed [15].

\section{Results}

\section{Demographic characteristics}

The participants' characteristics are presented in Table 1. The 36 healthy participants (male $=23$, female $=13$ ) had a mean age of $26.36 \pm 5.57$ years, mean weight of $62.19 \pm 8.09$ $\mathrm{kg}$, mean height of $169.02 \pm 7.46 \mathrm{~cm}$, mean BMI of $21.74 \pm$ $2.23 \mathrm{~kg} / \mathrm{m}^{2}$, and mean upper arm circumference of $37.24 \pm$ $3.09 \mathrm{~cm}$.

\section{Intra-rater reliability analysis}

A summary of the results for the intra-rater reliability of the $\mathrm{BB}$ and $\mathrm{TB}$ muscle thickness for the two sessions performed by two raters is shown in Table 2. The ICCs for intra-rater reliability ranged from 0.887 to 0.989 and the CI was within an acceptable range of 0.778 to 0.994 . The SEM values ranged from 0.303 to 0.866 and the MDC values ranged from 0.84 to 2.40 .

\section{Inter-rater reliability analysis}

The ICCs for inter-rater reliability ranged from 0.758 to 0.925 (Table 3). The SEM values ranged from 0.702 to 1.486 and the MDC values ranged from 1.95 to 4.12 .

\section{Discussion}

The aim of the study was to investigate the intra- and inter-rater reliability in obtaining D-RUSI muscle thickness measurements of the $\mathrm{BB}$ and $\mathrm{TB}$ muscles at rest and during the co-activation state. The D-RUSI is the only instrument that can assess and measure two muscles at the same time with two probes; therefore, the results cannot be directly compared with previous studies.

In this study, the D-RUSI of the BB muscle had very good

Table 1. Characteristics of the study participants $\quad(\mathrm{N}=36)$

\begin{tabular}{lc}
\multicolumn{1}{c}{ Parameter } & \multicolumn{1}{c}{ Value } \\
\hline Sex (male/female) & $23 / 13$ \\
Age $(\mathrm{yr})$ & $26.36(5.57)$ \\
Height $(\mathrm{cm})$ & $169.02(7.46)$ \\
Weight $(\mathrm{kg})$ & $62.19(8.09)$ \\
Body mass index $\left(\mathrm{kg} / \mathrm{m}^{2}\right)$ & $21.74(2.23)$ \\
Upper arm circumference $(\mathrm{cm})$ & $37.24(3.09)$ \\
\hline
\end{tabular}

Values are presented as number only or mean (SD). 
Table 2. Intra-rater reliability between repeated measures on D-RUSI for BB and TB muscle thickness (unit: mm) (N=36)

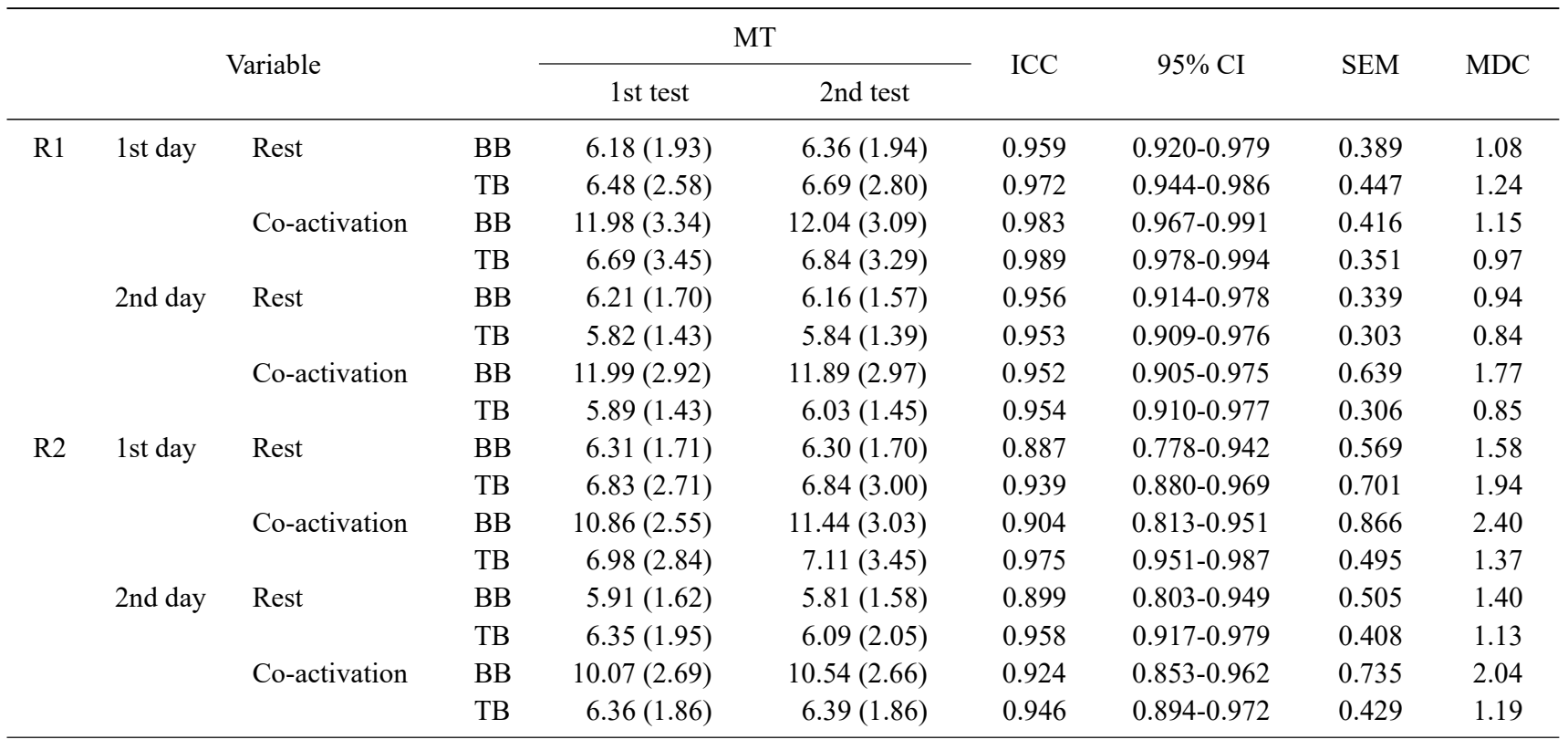

Values are presented as mean (SD).

D-RUSI: dual rehabilitative ultrasound imaging, BB: biceps brachii, TB: triceps brachii, MT: muscle thickness, ICC: intra-class correlation coefficient, 95\% CI: 95\% confidence interval, SEM: standard error of the measurement, MDC: minimal detectable change, R1: rater 1, R2: rater 2.

Table 3. Inter-rater reliability between repeated measures on D-RUSI for BB and TB muscle thickness (unit: mm)

$(\mathrm{N}=36)$

\begin{tabular}{|c|c|c|c|c|c|c|c|}
\hline \multirow{2}{*}{ Condition } & \multirow{2}{*}{ Muscle } & \multicolumn{2}{|c|}{ MT } & \multirow{2}{*}{ ICC } & \multirow{2}{*}{$95 \% \mathrm{CI}$} & \multirow{2}{*}{ SEM } & \multirow{2}{*}{ MDC } \\
\hline & & $\mathrm{E} 1^{\mathrm{a}}$ & $\mathrm{E} 2^{\mathrm{a}}$ & & & & \\
\hline \multirow[t]{2}{*}{ Rest } & $\mathrm{BB}$ & $6.27(1.93)$ & $6.30(1.69)$ & 0.849 & $0.759-0.906$ & 0.702 & 1.95 \\
\hline & $\mathrm{TB}$ & $6.59(2.67)$ & $6.83(2.83)$ & 0.925 & $0.881-0.953$ & 0.753 & 2.09 \\
\hline \multirow[t]{2}{*}{ Co-activation } & BB & $12.01(3.19)$ & $11.155(2.79)$ & 0.758 & $0.614-0.849$ & 1.486 & 4.12 \\
\hline & TB & $6.77(3.35)$ & $7.04(3.14)$ & 0.879 & $0.807-0.925$ & 1.125 & 3.12 \\
\hline
\end{tabular}

Values are presented as mean (SD).

D-RUSI: dual rehabilitative ultrasound imaging, BB: biceps brachii, TB: triceps brachii, MT: muscle thickness, ICC: intra-class correlation coefficient, 95\% CI: 95\% confidence interval, SEM: standard error of the measurement, MDC: minimal detectable change, E1: examiner 1, E2: examiner 2 .

${ }^{a}$ Based on three images taken by the examiner on the same day (day 1 ).

intra-rater reliability $(\mathrm{R} 1$ rest $\mathrm{ICC}=0.956-0.959, \mathrm{R} 1 \mathrm{co}-\mathrm{acti}-$ vation $\mathrm{ICC}=0.952-0.983$, R2 rest $\mathrm{ICC}=0.887-0.899, \mathrm{R} 2$ co-activation ICC $=0.904-0.924$; Table 2), very good inter-rater reliability at rest $(\mathrm{ICC}=0.849)$, and good inter-rater reliability at co-activation ( $\mathrm{ICC}=0.758$; Table 3 ). Our data on BT thickness were similar to those reported in a previous study, in which the thickness of the biceps tendon was measured twice in the same posture as in this study and showed very good intra- and inter-rater reliability (ICC $=0.98-0.99$ ) [16]. Although the measurement position was different, another study showed very good reliability $(\mathrm{ICC}=0.90)$ of biceps thickness measurement [17]. The biceps muscle is rela- tively easy to capture in ultrasound imaging because it is located in the superficial and the anterior layer relative to the position of the humerus [16]. It is believed that this factor contributed to obtaining the high reliability results. The comparison that resulted in the lowest reliability rating for BT thickness was the inter-rater reliability for co-activation. Muscle contraction can be influenced by many factors such as the evaluator's instructions, the motivation of the participant, and the skill of the participant at the time of motor control, all of which could explain the degradation of reliability. However, regardless of where the specific bias occurs, the actual difference between the measurements was small and 
did not cause a significant reduction in reliability.

ICC values showed very good intra-rater reliability (R1 rest $\mathrm{ICC}=0.953-0.972, \mathrm{R} 1$ co-activation $\mathrm{ICC}=0.954-0.989$, $\mathrm{R} 2$ rest $\mathrm{ICC}=0.939-0.958$, $\mathrm{R} 2$ co-activation $\mathrm{ICC}=0.946$ 0.975 ) and inter-rater reliability (rest $\mathrm{ICC}=0.925$, co-activation ICC $=0.879$ ) for the TB muscle (Tables 2, 3). In a previous study, Thoirs and English [18] measured the thickness of several muscle groups by ultrasonography in various postures. Although the measurement posture differed from that in the present study, their intra-examiner reliability was also very good when measuring the thickness of the anterior and posterior upper arm. In the present study, the reliability of the TB measurement was high and the ICC value for the co-activation state was lower than the ICC value for the resting state, for similar reasons as the BB measurement. Overall, D-RUSI measurement can be considered effective in the context of co-activation of the $\mathrm{BB}$ and $\mathrm{TB}$ muscles.

We also calculated the SEM and MDC values in order to assess measurement errors between repeated measurements and to determine whether changes occurred between these iterations. The SEM value can be used as an estimate for the entire group based on the CI. For example, the SEM value of the $\mathrm{BB}$ results measured by the first rater in the resting state on the first day was calculated as [observed score \pm 1.96 (SEM)]. Using this calculated value, there is a 95\% probability that the actual average score of the group will be between $5.42 \mathrm{~mm}$ and $6.94 \mathrm{~mm}$ when the group average is 6.18 $\mathrm{mm}$. The MDC value was calculated to range from $0.84 \mathrm{~mm}$ to $4.12 \mathrm{~mm}$. This suggests that we can expect $95 \%$ of patients without changes in this population to demonstrate a random variation of less than 0.84 to $4.12 \mathrm{~mm}$ in a repeat measurement of the D-RUSI [19].

There are several limitations to this study. First, because the experiment was conducted with a limited number of participants, measurement postures, and ranges, the results may not be generalized. Second, only the co-activation state with the BB acting as an agonist and TB acting as an antagonist was measured. Further studies should continue to investigate the reliability of the D-RUSI measures; in particular, an investigation of the changes in muscle thickness with TB acting as an agonist and BB acting as an antagonist may increase the reliability of the D-RUSI as a tool to measure muscle action during co-activation of the upper arm.

In conclusion, the D-RUSI is a useful tool for the simultaneous measurement of the thickness of two muscles when the $\mathrm{BB}$ is an agonist and the $\mathrm{TB}$ is an antagonist during co-activation of the upper arm.

\section{Conflict of Interest}

The authors declared no potential conflicts of interest with respect to the authorship and/or publication of this article.

\section{References}

1. Liu CJ, Becher J, Ford S, Heine K, Scheidt E, Wilson A. Effects of upper-extremity progressive resistance strength training in older adults: the missing picture. Phys Occup Ther Geriatr 2011; 29:255-69.

2. Kraemer WJ, Adams K, Cafarelli E, Dudley GA, Dooly C, Feigenbaum MS, et al. American college of sports medicine position stand. Progression models in resistance training for healthy adults. Med Sci Sports Exerc 2002;34:364-80.

3. Lindle RS, Metter EJ, Lynch NA, Fleg JL, Fozard JL, Tobin J, et al. Age and gender comparisons of muscle strength in 654 women and men aged 20-93 yr. J Appl Physiol (1985) 1997;83: 1581-7.

4. McGee CW, Mathiowetz V. The relationship between upper extremity strength and instrumental activities of daily living performance among elderly women. OTJR: Occupation, Participation and Health 2003;23:143-54.

5. MacKenzie SJ, Rannelli LA, Yurchevich JJ. Neuromuscular adaptations following antagonist resisted training. J Strength Cond Res 2010;24:156-64.

6. Petrofsky J, Batt J, Suh HJ, Jones R, Ushak N, Tucker JP, et al. Muscle use during isometric co-contraction of agonist-antagonist muscle pairs in the upper and lower body compared to abdominal crunches and a commercial multi gym exerciser. J Appl Res 2006;6:300-28.

7. Billot M, Simoneau E, Van Hoecke J, Martin A. Coactivation at the ankle joint is not sufficient to estimate agonist and antagonist mechanical contribution. Muscle Nerve 2010;41:511-8.

8. Hall L, Tsao H, MacDonald D, Coppieters M, Hodges PW. Immediate effects of co-contraction training on motor control of the trunk muscles in people with recurrent low back pain. J Electromyogr Kinesiol 2009;19:763-73.

9. Maeo S, Yoshitake Y, Takai Y, Fukunaga T, Kanehisa H. Effect of short-term maximal voluntary co-contraction training on neuromuscular function. Int J Sports Med 2014;35:125-34.

10. Schrank EC, Abraham K, Wilson MJ, Myers WV, King MG. The reliability of rehabilitative ultrasound imaging measurements of the lumbar multifidi recorded by physical therapist students with minimal training. J Phys Ther Educ 2011;25:57-62.

11. Matta T, Simão R, de Salles BF, Spineti J, Oliveira LF. Strength training's chronic effects on muscle architecture parameters of different arm sites. J Strength Cond Res 2011;25:1711-7.

12. Hodges PW, Pengel LH, Herbert RD, Gandevia SC. Measurement of muscle contraction with ultrasound imaging. Muscle Nerve 2003;27:682-92.

13. Miyatani M, Kanehisa H, Ito M, Kawakami Y, Fukunaga T. The accuracy of volume estimates using ultrasound muscle thickness measurements in different muscle groups. Eur J Appl Physiol 2004;91:264-72. 
14. Cho KH, Lee HJ, Lee WH. Reliability of rehabilitative ultrasound imaging for the medial gastrocnemius muscle in poststroke patients. Clin Physiol Funct Imaging 2014;34:26-31.

15. Koppenhaver SL, Hebert JJ, Fritz JM, Parent EC, Teyhen DS, Magel JS. Reliability of rehabilitative ultrasound imaging of the transversus abdominis and lumbar multifidus muscles. Arch Phys Med Rehabil 2009;90:87-94.

16. Drolet P, Martineau A, Lacroix R, Roy JS. Reliability of ultrasound evaluation of the long head of the biceps tendon. J Rehabil Med 2016;48:554-8.
17. Ohya N, Yamada T, Satoh Y, Kawamura H. Relative and absolute reliability of ultrasound measurements for the thickness of the soft tissue around the shoulder joint of young normal subjects. J Phys Ther Sci 2017;29:754-9.

18. Thoirs K, English C. Ultrasound measures of muscle thickness: intra-examiner reliability and influence of body position. Clin Physiol Funct Imaging 2009;29:440-6.

19. Portney LG, Watkins MP. Foundations of clinical research: applications to practice. 3rd ed. New Jersey: Pearson Education; 2009. 